\title{
$\gamma$-Ray Bursts and Afterglows from Rotating Strange Stars and Neutron Stars
}

\author{
Z. G. Dai and T. Lu \\ Department of Astronomy, Nanjing University, Nanjing 210093, China
}

\begin{abstract}
We here discuss a new model of $\gamma$-ray bursts (GRBs) based on differentially rotating strange stars. Strange stars in this model and differentially rotating neutron stars in the KluźniakRuderman model can produce extremely relativistic, variable fireballs required by GRBs and then become millisecond pulsars. The effect of such pulsars on expansion of the postburst fireballs through magnetic dipole radiation is studied. We show that these two models can explain naturally not only various features of GRBs but also light curves of afterglows.
\end{abstract}

PACS numbers: 97.70.Rz, 12.38.Mh, 26.60.+c, 97.60.Jd

$\gamma$-ray bursts (GRBs) has been one of the greatest mysteries in high-energy astrophysics for about thirty years. The observations of GRBs and a few afterglows [1-3] show that their sources are at cosmological distances. These observations require that any energysource model should satisfy the following general features: (i) The model should produce an extremely relativistic fireball containing an amount of energy $\approx 10^{51}-10^{52}$ ergs [4]. (ii) The high Lorentz factor of the fireball implied by the short variability and the nonthermal spectra [5] requires that the fraction of contaminating baryons be less than $1 \%$ of the explosive energy (viz., $\leq 10^{-5} M_{\odot}$ ). (iii) The fireball is highly variable (required by the multi-peak feature of the light curves of GRBs) and it should last for the GRB duration (typically a few dozen seconds), implying that the source cannot release energy only once [4,6]. (iv) The fireball should be a rare event occurring about once per million years in a galaxy [4]. (v) Finally, the model should account for the decline-rise-decline behavior of the R-band light curve of the afterglow from GRB $970508[7,8]$. In this Letter, we combine features of some existing pulsar GRB models, build upon them, and synthesize 
a new model of GRBs based on differentially rotating strange stars.

Recently Kluźniak and Ruderman [9] argued differentially rotating neutron stars as an origin of GRBs: In a differentially rotating neutron star, internal poloidal magnetic field $(B)$ will be wound up into a toroidal configuration and linearly amplified as one part of the star rotates about the other part. Only when it increases up to a critical field value, $B_{f}$, will the toroidal field be sufficiently buoyant to overcome fully the stratification in neutron star composition. And then the buoyant magnetic torus will be able to float up to break through the stellar surface. Reconnection of the newborn surface magnetic field will lead to a quickly explosive event with a large amount of energy, which could be a sub-burst of a GRB. This model accounts well for the typical fluence in each observed sub-burst (peak), for the number of peaks, for the time interval between peaks, and for the rapid rise times and variability. This model may result in low-mass loading baryonic matter.

Now let's discuss a new model of GRBs based on rotating strange stars in detail. Strange stars have been widely studied $[10,11]$. However, two objections against the existence of isolated strange stars (with mass $\sim 1.4 M_{\odot}$ ) result from astrophysical arguments. On the one hand, it has been argued that the disruption of a single strange star can contaminate the entire galaxy, and thus all neutron stars have in fact converted to strange stars [12]. Furthermore, if strange stars can be created directly in supernovae, then some strange stars must have more massive compact companions, and merging of such binaries will lead to the Madsen-Caldwell-Friedman effect [13]. This result conflicts with the postglitch behavior of pulsars which has been described well by the neutron-superfluid vortex creep theory, rather than by the strange-star models [14]. On the other hand, an isolated neutron star with a stiff equation of state $\left(\right.$ EOS) and with mass of $\sim 1.4 M_{\odot}$ cannot convert to a strange star, because its central density is lower than the deconfinement density of neutron matter $\sim(6-9) \rho_{0}$ (here $\rho_{0}$ is the nuclear-matter density) [15].

However, there are two scenarios by which strange stars are born, and which don't conflict with the above two arguments. First, it has been proposed [16] that when neutron 
stars in low-mass x-ray binaries accrete sufficient mass, they may convert to strange stars. The conversion should proceed in a detonation mode (at the speed of sound) [17], and thus the timescale for this process is about $0.1 \mathrm{~ms}$. The direct criterion for the conversion is that the total masses of accreting neutron stars exceed $\sim 1.8 M_{\odot}$ if the EOS at high density is rather stiff. Fortunately, the theoretical analysis shows that the amount of matter accreted by the radio pulsars in many binary systems exceeds $\sim 0.5 M_{\odot}[18]$. If this is true, some of these pulsars should have masses over $1.8 M_{\odot}$. The observation of $\mathrm{kHz}$ quasi-periodic oscillations in eight low-mass x-ray binaries and the analysis of highresolution optical spectroscopy of Cyg X-2 indicate that the masses of neutron stars in these systems are larger than $1.8 M_{\odot}[19]$.

We consider an alternative scenario. It is usually thought that due to gravitational radiation a neutron-star binary will eventually merge to a black hole. This results from the argument that the EOS for neutron matter at high density is soft. But, the EOSs in neutron stars are thought to be moderately stiff to stiff for the following reasons: (i) Soft EOSs at high densities are ruled out by the postglitch recovery in four pulsars [20]. (ii) The high masses $\left(\geq 1.8 M_{\odot}\right)$ of the neutron stars in a few low-mass $x-r a y$ binaries also rule out soft EOSs [19]. (iii) The soft EOS such as kaon condensation doesn't seem to occur in stable neutron stars [21]. If the EOS for neutron matter is sufficiently stiff, therefore, the post-merger objects of Hulse-Taylor-like binaries may be massive neutron stars rather than black holes. The same outcome would be achieved if the initial masses of the merging neutron stars were low, e.g. $M \sim 1 M_{\odot}$. According to the first scenario, these massive neutron stars will subsequently convert to strange stars.

If strange stars form in some x-ray binaries by these scenarios, we now show that such stars are very difficult to be disrupted. The total number of strange stars in a galaxy is about $10^{4}$ and their number in globular clusters (GCs) is $N_{s s} \sim 10^{3}$. The neutron star number density in GCs is $n_{n s} \sim 3 \cdot 10^{-59}\left(R_{g} / 10 \mathrm{kpc}\right)^{-3} \xi \mathrm{cm}^{-3}$, where $R_{g}$ is the galactic radius and $\xi \sim 10$, and we have assumed that the total neutron-star number is $\sim 10^{8}$. The cross-section for a neutron star capturing a strange star is usually approximated by $\sigma \sim 4 \pi G^{2} M^{2} / v^{4}$, where $M$ is the mass of the neutron star and $v$ its 
velocity. Therefore, we find the rate for neutron star-strange star capture events in GCs, $\Re \sim \sigma v n_{n s} N_{s s} \sim 10^{-17}\left(M / M_{\odot}\right)^{2}\left(v / 500 \mathrm{~km} \mathrm{~s}^{-1}\right)^{-3}\left(R_{g} / 10 \mathrm{kpc}\right)^{-3}(\xi / 10) \mathrm{yr}^{-1}$, and the rate outside GCs should be smaller. The actual rate for the strange stars to be first captured and then disrupted should be much less than $\Re$ due to the requirement of massive neutron stars whose number is rather small.

For the above two scenarios, the pre-conversion neutron stars all have periods of the order of $1 \mathrm{~ms}$ because of accretion-induced spin-up in low-mass x-ray binaries and synchrotronization during the coalescence of neutron-star binaries. Since the moments of inertia always decrease during the conversion, the resulting strange stars will rotate at periods $\leq 1 \mathrm{~ms}$. Furthermore, differential rotation may occur in the interiors of these newborn strange stars due to the fact that the density profile of a strange star is much different from that of a neutron star with the same mass [11]. According to the basic idea of the Kluźniak-Ruderman model, such differentially rotating strange stars could lead to a series of sub-bursts of GRBs.

Our model can account for the following features. First, sufficient energy. The total available energy released in our model should be the differential rotation energy, viz., $E=\hat{I} \Omega^{2} / 2=5 \cdot 10^{51} \operatorname{ergs} \hat{I}_{44} \Omega_{4}^{2}$, where $\hat{I}$ is the effective moment of inertia of differential rotation $\left(\hat{I}_{44}=\hat{I} / 10^{44} \mathrm{~g} \mathrm{~cm}^{2}\right)$, and $\Omega$ the effective difference in angular velocity of rotation $\left(\Omega_{4}=\Omega / 10^{4} \mathrm{~s}^{-1}\right)$. We here estimate the critical field based on $B_{f}^{2} /(8 \pi)=f \rho c_{s}^{2}$, where $f$ is the dimensionless parameter accounting for the stratification $(\sim 1 \%$ for massive strange stars), $\rho$ the typical mass density of the strange star, and $c_{s}$ the speed of sound $\left(c_{s}=c / \sqrt{3}\right.$ for strange matter $)$. Thus, we obtain $B_{f} \approx 2 \cdot 10^{17}\left(\rho / 4 \cdot 10^{14} \mathrm{~g} \mathrm{~cm}^{-3}\right)^{1 / 2} \mathrm{G}$.

Second, very-low-mass baryon contamination. Because the strange star has a thin crust $\left(\sim 10^{-5} M_{\odot}\right)[10,11]$, the resulting fireball in our model is almost free of baryons, alleviating the baryon-contamination problem. This point was raised by Haensel et al. [22], who considered merging of strange stars as an origin of GRBs. Of course, this problem can also be avoided in many models [23-25], where power is extracted from rapidly spinning neutron stars or black holes with strong magnetic fields by Poynting 
flux, which are later converted to $\gamma$-rays and lower-frequency photons. But, it is unclear whether these models can also explain the spiky light curves of GRBs.

Third, temporal property. Following [9], we estimate the time interval between subbursts, $\tau_{i}=(2 \pi / \Omega)\left(B_{f} / B\right) \approx 10 \mathrm{~s} B_{13}^{-1} \Omega_{4}^{-1}$, and the total duration, $\tau_{d} \approx 60 \mathrm{~s} B_{13}^{-1} \Omega_{4}$, where $B_{13}$ is in units of $10^{13} \mathrm{G}$. The internal field could be generated by either field-freezing or by a dynamo. Duncan and Thompson [23] have shown that whether a dynamo operates due to convection driven by a large neutrino flux is dependent on the Rossby number $N_{R}=P / \tau_{\text {conv }}$, where $P$ is the stellar period and $\tau_{\text {conv }}$ the convection overturn timescale at the base of the convection zone. If $N_{R} \leq 1$, an efficient dynamo can result and the (poloidal) field grows exponentially to $B \sim 10^{15} \mathrm{G}$; if $N_{R}>1$, a dynamo is suppressed. It means that if a dynamo has operated, the duration is $\tau_{d} \approx 0.6 \mathrm{~s}\left(B_{13} / 10^{2}\right)^{-1} \Omega_{4}$, leading to short bursts; but if not, long bursts will form. This provides an explanation for the bimodal duration distribution of GRBs. This distribution has also been proposed to be associated with submillisecond neutron stars through gravitational instability [25].

Fourth, low burst rate. It has been argued $[16,26]$ that the rate of conversion of accreting neutron stars in low-mass x-ray binaries, $\sim 10^{-6} /$ yr per galaxy, is consistent with the observed GRB rate. In addition, the rate of coalesence of Hulse-Taylor-like binaries has been shown to correspond closely to the observed GRB rate [27].

Both the Kluźniak-Ruderman model and our model can produce an extremely relativistic, variable fireball, which will lead to internal shocks [28] and subsequent external shocks [29]. A GRB will be produced once the kinetic energy is dissipated and radiated as $\gamma$-rays through synchrotron or possibly inverse-Compton emission from the accelerated electrons in these shocks. At the same time, the strange star is becoming a pulsar. Therefore, two common by-products of these models are a strongly magnetic millisecond pulsar and a postburst relativistic fireball. It is natural to expect that the central pulsar affects the evolution of the postburst fireball and the afterglow from this fireball. Can this effect explain the observed afterglows? Yes. Here we would provide a case in which this effect can explain well the optical afterglow of GRB 970508. 
At the center of the fireball, the pulsar loses its rotational energy through magnetic dipole radiation, whose power varies with time as $L(t) \propto(1+t / T)^{-2}$, where $t$ is one measure of time in the burster's rest frame, and $T$ the initial spin-down timescale. For $t<T, L$ can be thought as a constant; but for $t \gg T, L$ decays as $\propto t^{-2}$.

The pulsar radiates electromagnetic waves with frequency of $\omega=2 \pi / P \sim 10^{4} \mathrm{~s}^{-1}$. These waves are absorbed by the shocked interstellar medium (ISM) because the plasma frequency of the shocked ISM is much higher than $\omega$. This implies that the pulsar pumps continuously its rotational energy into the shocked ISM. We assume that the expansion of the fireball in uniform ISM is relativistic and adiabatic. At a time $t$, the shocked ISM energy is given by $4 \pi r^{2}\left(r / 4 \gamma^{2}\right) \gamma^{2} e^{\prime}$, where $r \approx c t$ is the blastwave radius, $\gamma$ the Lorentz factor of the fireball, and $e^{\prime}=4 \gamma^{2} n m_{p} c^{2}$ the shocked ISM energy density in the comoving frame (where $n$ is the electron number density of the unshocked ISM) [30]. This energy should be equal to the sum of half of the initial energy $(E / 2)$ and the energy which the fireball has obtained from the pulsar based on energy conservation:

$$
4 \pi n m_{p} c^{2} \gamma^{2} r^{3}=\frac{E}{2}+\int_{0}^{t}(1-\beta) L(t-r / c) d t
$$

where $\beta=\left(1-1 / \gamma^{2}\right)^{1 / 2}$. Because the Lorentz factor of the fireball at the initial stage decays as $\gamma \propto t^{-3 / 2}$, the timescale in which the fireball has obtained energy $\sim E / 2$ from the pulsar is estimated as $4 \gamma^{2} E / L$, measured in the burster's rest frame. The corresponding observer-frame timescale $(\tau)$ is equal to this timescale divided by $2 \gamma^{2}$, viz., $\tau=2 E / L$. We assume $\tau \ll T$, or $P<1.6 \mathrm{~ms}\left(E_{51} / 4\right)^{-1 / 2} I_{45}^{1 / 2}$. Clearly, this inequality is independent on the stellar magnetic field. Please note that Blackman and Yi [31] recently discussed the dissipation of the rotational energy of a pulsar by large amplitude electromagnetic waves, which are directly converted to an afterglow, but in our model, afterglows result from shocks absorbing such waves.

We consider below the synchrotron radiation from the accelerated electrons behind the shock, and assume a power-law distribution of the electrons with index $p$. We analyze evolution of the afterglow: (i) At the initial stage, viz., observer-frame time $t_{\oplus} \ll \tau$, the second term on the right side of equation (1) can be neglected and the Lorentz factor of the 
fireball decays as $\gamma \propto t_{\oplus}^{-3 / 8}$. In this case, we have found that the fireball will rapidly go into the slow cooling phase, and the radiation at optical band will come from those radiative electrons a few hours later after the initial burst. This conclusion is similar to that of the recent studies [32,33]. Therefore, the optical flux from the fireball $F_{\nu} \propto \nu^{-p / 2} t_{\oplus}^{(2-3 p) / 4}$. The result $p \approx 1.0$ inferred from the observed spectrum index in two days following the initial burst [8] leads to the R-band flux $\propto t_{\oplus}^{-1 / 4}$. This satisfactorily accounts for the slow decline of the R-band light curve in $\sim 8$ hours after the burst [8].

(ii) For $T>t_{\oplus} \gg \tau$, the term $E / 2$ in equation (1) can be neglected and the Lorentz factor of the fireball decays as $\gamma \propto t_{\oplus}^{-1 / 4}$. In this case, the comoving-frame equipartition magnetic field decreases as $B^{\prime} \propto \gamma \propto t_{\oplus}^{-1 / 4}$, and the synchrotron break frequency drops in time as $\nu_{m} \propto \gamma^{3} B^{\prime} \propto t_{\oplus}^{-1}$. At the same time, since the comoving electron number density is $n_{e}^{\prime} \propto \gamma \propto t_{\oplus}^{-1 / 4}$ and the comoving width of the emission region $\Delta r^{\prime} \sim r / \gamma \propto t_{\oplus}^{3 / 4}$, according to Mészáros \& Rees in [7], the comoving intensity $I_{\nu}^{\prime} \propto n_{e}^{\prime} B^{\prime} \Delta r^{\prime} \propto t_{\oplus}^{1 / 4}$. So the observed peak flux density increases in time based on $F_{\nu_{m}} \propto t_{\oplus}^{2} \gamma^{5} I_{\nu_{m}}^{\prime} \propto t_{\oplus}$. For a power-law distribution, electrons with different Lorentz factors may have different radiative efficiencies. Sari et al. [32] have given a critical value, $\gamma_{c} \propto \gamma^{-3} t_{\oplus}^{-1} \propto t_{\oplus}^{-1 / 4}$, above which cooling by synchrotron radiation is significant. The corresponding synchrotron frequency decays as $\nu_{c} \propto \gamma B^{\prime} \gamma_{c}^{2} \propto t_{\oplus}^{-1}$. From [32], we find the optical flux $F_{\nu}=\left(\nu_{c} / \nu_{m}\right)^{-(p-1) / 2}\left(\nu / \nu_{c}\right)^{-p / 2} F_{\nu_{m}} \propto \nu^{-p / 2} t_{\oplus}^{(2-p) / 2}$, and therefore, $p \approx 1.0$ further leads to the result that the optical flux increases with time, being in agreement with the subsequent rise of the R-band light curve [8].

(iii) For $t_{\oplus} \gg T$, the power of the pulsar due to magnetic dipole radiation rapidly decreases as $L \propto t^{-2}$, and the evolution of the fireball is hardly influenced by the stellar radiation. As in stage (i), the optical flux is $F_{\nu} \propto \nu^{-p / 2} t_{\oplus}^{(2-3 p) / 4}$. Because $p \approx 2.2$, inferred from the spectrum index observed two days later after the burst, the R-band flux $\propto t_{\oplus}^{-1.2}$, which is also well consistent with the observations [8].

The effect of millisecond pulsars on afterglows can also be shown schematically in Figure 1. We further give constraints on stellar parameters. According to the definitions of $\tau$ 
and $T$, we obtain the stellar period, $P=0.6 \mathrm{~ms}\left(E_{51} / 4\right)^{-1 / 2}(T / 2 \mathrm{~d})^{-1 / 2}(\tau / 0.4 \mathrm{~d})^{1 / 2} I_{45}^{1 / 2}$, and the magnetic field strength $B_{13}=3.1\left(E_{51} / 4\right)^{-1 / 2}(T / 2 \mathrm{~d})^{-1}(\tau / 0.4 \mathrm{~d})^{1 / 2} I_{45} R_{6}^{-3}$, where $E_{51}=$ $E / 10^{51} \mathrm{ergs}, I_{45}$ is the moment of inertia in $10^{45} \mathrm{~g} \mathrm{~cm}^{2}$ and $R_{6}$ the stellar radius in $10^{6} \mathrm{~cm}$. The observations indicate $\tau \sim 0.4$ days and $T \sim 2$ days [8]. If taking $\left(E_{51} / 4\right)^{-0.5} I_{45} R_{6}^{-3} \sim$ 1 , we have $B_{13} \sim 3.1$, and the total duration of the main burst $\left(t_{d}\right)$ is estimated to be about 20 seconds. This is in excellent agreement with the observation by the BeppoSAX satellite [34]. In addition, we find $P \approx 0.6 \mathrm{~ms}$, implying that the pulsar has a submillisecond period. What we want to point out is that such a period for the formation scenario of a strange star in a low-mass x-ray binary may be due to preconversion accretion-induced spin-up and due to decrease of stellar moment of inertia during conversion, and a magnetic field $\sim 10^{13} \mathrm{G}$ may result from preconversion crustal plate motion and/or from a dynamo in the strange star.

If a GRB results from magnetic reconnection on the surface of a differentially rotating strange star or neutron star, we cannot clearly determine the region in which such a process takes place. A beaming of the emission might form if this process occurs on a small area of the stellar surface as solar flares. This might provide a solution to the large-energy problem of GRB 971214.

Finally, it is interesting to note that two other kinds of high-energy transients, the soft $\gamma$-ray repeaters and the hard x-ray burster (GRO J1744-28), have also been described well by the strange-star models in which the stellar crust-breaking leads to bursts [35].

We thank the referees for their enlightening comments and valuable suggestions. This work was supported by the National Natural Science Foundation of China.

\section{References}

[1] G. J. Fishman and C. A. Meegan, Ann. Rev. Astron. Astrophys. 33, 415 (1995).

[2] J. van Paradijs et al., Nature (London) 386, 686 (1997).

[3] M. R. Metzger et al., Nature (London) 387, 878 (1997).

[4] For a recent review see T. Piran, astro-ph/9801001. 
[5] E. Woods and A. Loeb, Astrophys. J. 453, 583 (1995).

[6] R. Sari and T. Piran, Astrophys. J. 485, 270 (1997).

[7] Simple analytical models are successful at explaining the overall power-law decay behavior of light curves [e.g., P. Mészáros and M. J. Rees, Astrophys. J. 476, 232 (1997); E. Waxman, ibid. 485, L5 (1997); M. Vietri, ibid. 488, L105 (1997); R. Wijers, M. J. Rees and P. Mészáros, Mon. Not. R. Astron. Soc. 288, L51 (1997)]. But, the optical and x-ray light curves of the observed afterglows have been shown to depart from this behavior [8].

[8] S. G. Djorgovski et al., Nature (London) 387, 876 (1997); A. J. Castro-Tirado, et al., Science 279, 1011 (1998); H. Pedersen et al., Astrophys. J. 496, 311 (1998); T. J. Galama et al., ibid. 497, L13 (1998).

[9] W. Kluźniak and M. Ruderman, Astrophys. J. 505, L113 (1998).

[10] C. Alcock, E. Farhi and A. Olinto, Astrophys. J. 310, 261 (1986); P. Haensel, J. L. Zdunik and R. Schaeffer, Astron. Astrophys. 160, 121 (1986); N. K. Glendenning and F. Weber, Astrophys. J. 400, 647 (1992); Y. F. Huang and T. Lu, Astron. Astrophys. 325, 189 (1997).

[11] N. K. Glendenning, Compact Stars: Nuclear Physics, Particle Physics and General Relativity, (Springer-Verlag, New York, 1997), pp.337-361.

[12] J. Madsen, Phys. Rev. Lett. 61, 2909 (1988); R. R. Caldwell and J. L. Friedman, Phys. Lett. B 264, 143 (1991).

[13] W. Kluźniak, Astron. Astrophys. 286, L17 (1994).

[14] M. A. Alpar, Phys. Rev. Lett. 58, 2152 (1987).

[15] G. Baym, in Neutron Stars: Theory and observation, J. Ventura and D. Pines, eds. (Kluwer, Dordrecht, 1991), p.21.

[16] K. S. Cheng and Z. G. Dai, Phys. Rev. Lett. 77, 1210 (1996).

[17] G. Lugones, O. G. Benvenuto and H. Vucetich, Phys. Rev. D 50, 6100 (1994).

[18] E. P. J. van den Heuvel and O. Bizaraki, Astron. Astrophys. 297, L41 (1995).

[19] W. Zhang, T. E. Strohmayer and J. H. Swank, Astrophys. J. 482, L167 (1997); P. Kaaret, E. C. Ford and K. Chen, ibid. 480, L27 (1997); J. Casares, P. Charles and E. Kuulkers, ibid. 493, L39 (1998).

[20] B. Link, R. I. Epstein and K. A. Van Riper, Nature (London) 359, 616 (1992).

[21] V. R. Pandharipande, C. J. Pethick and V. Thorsson, Phys. Rev. Lett. 75, 4567 (1995); Z. G. Dai and K. S. Cheng, Phys. Lett. B 401, 219 (1997). 
[22] P. Haensel, B. Paczyński and P. Amsterdamski, Astrophys. J. 375, 209 (1991).

[23] R. C. Duncan and C. Thompson, Astrophys. J. 392, L9 (1992).

[24] V. V. Usov, Nature (London) 357, 452 (1992); C. Thompson, Mon. Not. R. Astron. Soc. 270, 480 (1994); E. G. Blackman, I. Yi and G. B. Field, Astrophys. J. 473, L79 (1996); P. Mészáros and M. J. Rees, ibid. 482, L29 (1997).

[25] I. Yi and E. G. Blackman, Astrophys. J. 494, L163 (1998).

[26] K. S. Cheng and Z. G. Dai, Astrophys. J. 492, 281 (1998).

[27] B. Paczyński, Acta Astron. 41, 257 (1991); R. Narayan, T. Piran and A. Shemi, Astrophys. J. 379, L17 (1991); E. S. Phinney, ibid. 380, L17 (1991).

[28] M. J. Rees and P. Mészáros, Astrophys. J. 430, L93 (1994); B. Paczyński and G. $\mathrm{Xu}$, ibid. 427, 708 (1994).

[29] M. J. Rees and P. Mészáros, Mon. Not. R. Astron. Soc. 258, 41p (1992).

[30] R. D. Blandford and C. F. McKee, Phys. Fluids 19, 1130 (1976).

[31] E. G. Blackman and I. Yi, Astrophys. J. 498, L31 (1998).

[32] R. Sari, T. Piran and R. Narayan, Astrophys. J. 497, L17 (1998).

[33] A. Panaitescu, P. Mészáros and M. J. Rees, astro-ph/9801258.

[34] L. Piro et al., Astron. Astrophys. 331, L41 (1998).

[35] K. S. Cheng and Z. G. Dai, Phys. Rev. Lett. 80, 18 (1998); K. S. Cheng, Z. G. Dai, D. M. Wei and T. Lu, Science 280, 407 (1998).

\section{FIGURE CAPTION}

FIG. 1. Afterglow light curves with a pulsar (solid line) and without a pulsar (dashed line). 


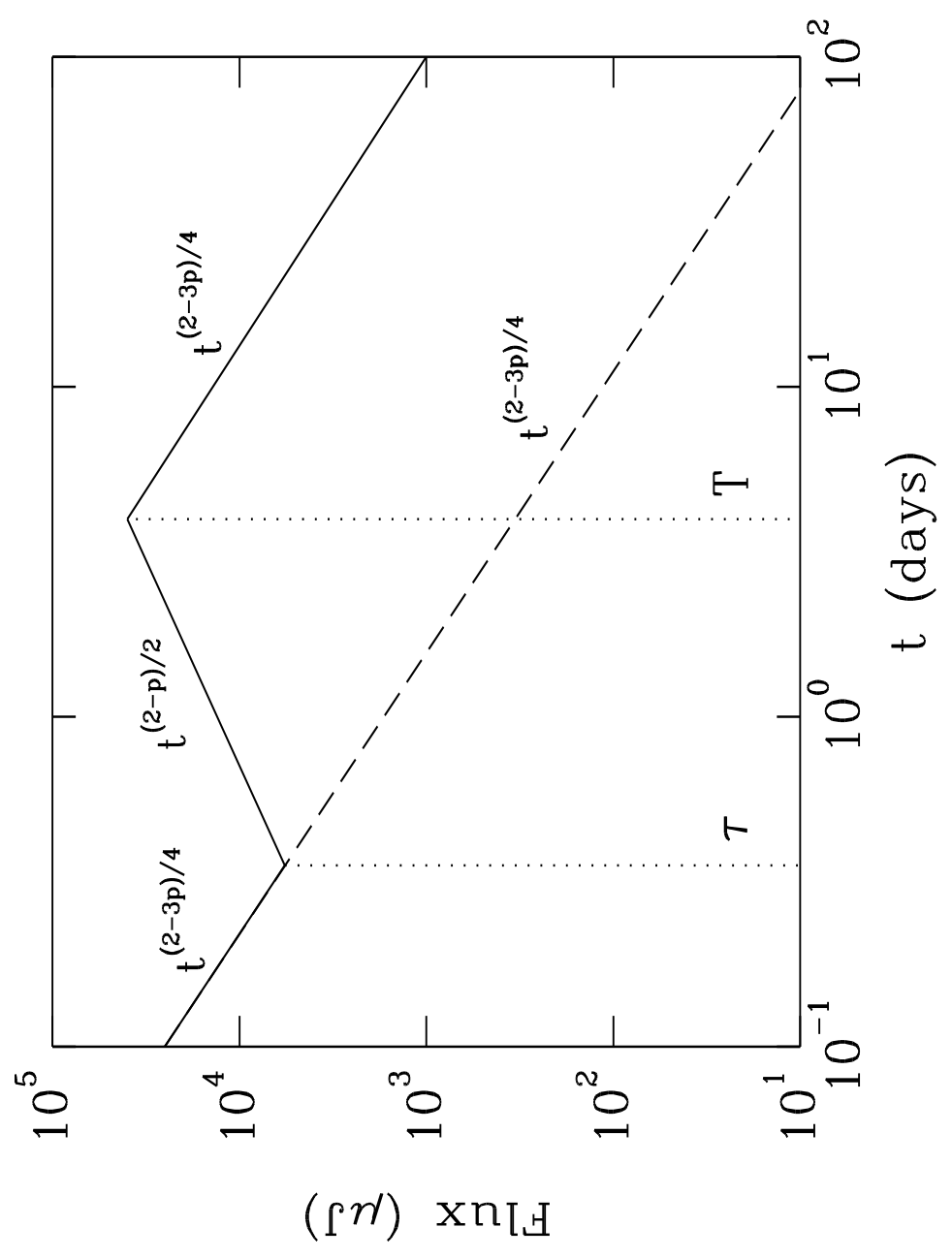

